\title{
Vascular density and phenotype around ductal carcinoma in situ (DCIS) of the breast
}

\author{
NB Teo', BS Shoker', C Jarvis', L Martin², JP Sloane', and C Holcombe ${ }^{*, 2}$ \\ 'Pathology Department, University of Liverpool, Duncan Building, Daulby Street, Liverpool L69 3GA, UK; ${ }^{2}$ Breast Unit, Linda McCartney Centre, Royal Liverpool \\ University Hospital, Prescot Street, Liverpool L7 8XP, UK
}

Up to $50 \%$ of recurrences of ductal carcinoma in situ of the breast are associated with invasive carcinoma but no pathological or molecular features have yet been found to predict for the development of invasive disease. For a tumour to invade, it requires the formation of new blood vessels. Previous studies have described a vascular rim around ducts involved by ductal carcinoma in situ, raising the possibility that the characteristics of periductal vascularisation may be important in determining transformation from in situ to invasive disease. Periductal vascular density and phenotype were determined using morphometry and a panel of anti-endothelial antibodies (von Willebrand factor, CD3I, CDI4I and CD34) and related to the presence of invasive carcinoma and other histological features. Compared to normal lobules, pure ductal carcinoma in situ exhibited a greater density of $\mathrm{CD}_{3} 4^{+}$and $\mathrm{CD} 31^{+}$vessels but a decrease in those that were immunopositive for $\mathrm{VWF}$, indicating a difference in phenotype and in density. Ductal carcinoma in situ associated with invasive carcinoma showed a profile of vascular immunostaining similar to that of pure ductal carcinoma in situ but there were significantly greater numbers of CD34 ${ }^{+}$ and $\mathrm{CDI} 4 \mathrm{I}^{+}$vessels and fewer staining for VWF. There was a significant negative correlation between vascular density and both the cross-sectional areas of the ducts involved and the extent of the necrosis of the tumour they contained. A correlation between vascular density and nuclear grade was also noted, being highest in the intermediate grade. The greater density of $\mathrm{CD}_{3} 4^{+}$and $\mathrm{CDI} 4 \mathrm{I}^{+}$vessels around ductal carcinoma in situ associated with invasive carcinoma could reflect a greater predisposition to invade but a direct effect of co-existent invasive carcinoma cannot entirely be ruled out in the present study. The relationship between vascular density, grade, duct size and nuclear grade suggests that periductal angiogenesis increases with tumour growth rate but is unable to keep pace with the most rapidly growing lesions. British Journal of Cancer (2002) 86, 905 -9l I. DOI: 10.1038/sj/bjc/6600053 www.bjcancer.com

(c) 2002 Cancer Research UK

Keywords: breast; ductal carcinoma in situ; angiogenesis; endothelial phenotype; invasive carcinoma

With the advent of screening mammography, the frequency with which ductal carcinoma in situ (DCIS) is detected has increased from approximately 1 to $20 \%$ of all breast cancers and is as high as 30\% in some centres (Nemoto et al, 1980; Fryberg and Bland, 1994). Histological grade, lesion size and excision margin status are strongly related to the probability of developing local recurrence (Recht et al, 1985; Silverstein et al, 1995; Badve et al, 1998) and important in determining management. Recurrent in situ carcinoma is itself innocuous but up to $50 \%$ of recurrences are associated with invasive carcinoma (Recht et al, 1985; Silverstein et al, 1995; Badve et al, 1998). To date, no pathological or molecular features have been found to predict for the development of invasive disease.

For a tumour to grow beyond $1-2 \mathrm{~mm}^{3}$, it requires the formation of new blood vessels to supply oxygen and nutrients as well as to excrete catabolites. There have been many studies of the process of angiogenesis in breast carcinoma but most have focused on invasive disease. In DCIS and other precursor lesions (Guidi et al, 1994; Engels et al, 1997a,b; Lee et al, 1997; Lee et al, 1999; Sales et al, 1999; Valtola et al, 1999), two patterns of

\footnotetext{
*Correspondence: C Holcombe; E-mail: cholcombe@rlbuhtr.nwest.nhs.uk ${ }^{3}$ Deceased.

Received I0 September 200I; accepted 5 November 200 I
}

vascularity have been described: diffuse stromal vascularity and a vascular rim around the involved ducts. Pre-malignant lesions of the breast can induce angiogenesis in animal experimental systems and in the human breast (Gimbrone and Gullino, 1976; Brem et al, 1977; Heffelfinger et al, 1996; Lichtenbeld et $a l, 1998)$, it thus seems possible that the pattern or extent of vascularisation around DCIS may be an important factor in determining the transformation from in situ to invasive carcinoma. It is likely that the periductal vessels are most important in this respect as incipient invasion is most likely to be associated with changes in vessels in the immediate vicinity of the tumour cells.

The main aim of this study was to test the hypothesis that the development of invasive carcinoma in DCIS is associated with changes in periductal vessels. The phenotype and number of microvessels were compared in pure DCIS with those in DCIS associated with invasive carcinoma. As studies of breast cancer angiogenesis in the past have been associated with inconsistent findings, we have used precise morphometric methodology to compare vascularity in DCIS with normal breast and have employed a panel of antiendothelial antibodies to take account of phenotypic as well as numerical changes. At the same time we have taken the opportunity to determine if vascular density and phenotype have any relationship to histological features, particularly nuclear grade, necrosis and duct size. 


\section{MATERIALS AND METHODS}

\section{Patients and tumours}

Formalin-fixed paraffin-embedded breast samples $(n=40)$ of patients with ductal carcinoma in situ, with $(n=20)$ and without $(n=20)$ invasive carcinoma, were collected from the archives of the Pathology Department of the Royal Liverpool University Hospital. The original haematoxylin and eosin (H\&E) stained sections of the primary tumours were reviewed by two pathologists (BS Shoker and JP Sloane) for classification by architecture and nuclear grade following the guidelines of the European Commission and UK National Breast Screening Programme (National coordinating group for breast screening pathology 1997, The consensus conference committee 1997). A representative block for each patient was selected for subsequent immunostaining.

\section{Immunohistochemistry}

Sections were stained for endothelial cells using monoclonal antiCD31 (JC/70A, DAKO, Denmark), anti-CD34 (Qbend/10, DAKO, Denmark), anti-CD141 (Anti-Thrombomodulin, DAKO, USA) and polyclonal anti-human von Willebrand factor (vWF) (DAKO, Denmark).

Sections were dewaxed through two changes of xylene and industrial methylated spirits (IMS). Endogenous peroxidase activity was blocked with a mixture of $\mathrm{H}_{2} \mathrm{O}_{2}$ /methanol $\left(12 \mathrm{ml} \mathrm{H}_{2} \mathrm{O}_{2}\right.$ in $400 \mathrm{ml}$ methanol) for $12 \mathrm{~min}$. Antigen retrieval was performed for the endothelial markers by treating the sections with $0.2 \mathrm{~g}$ of trypsin and $0.4 \mathrm{~g}$ of calcium chloride in $440 \mathrm{ml}$ Tris Buffered Saline (TBS (50 mM Tris- $\mathrm{HCl}, 150 \mathrm{~mm} \mathrm{NaCl}, \mathrm{pH} 7.4$ )) at $37^{\circ} \mathrm{C}$ for $20 \mathrm{~min}$. For Ki67 antigen retrieval was performed by immersing the sections in $2000 \mathrm{ml}$ of Ethylenediamine-Tetraacetic Acid (Sigma Chemical, St Louis USA) and then boiling in a pressure cooker for $8 \mathrm{~min}$. Prior to staining with the polyclonal antiserum, sections were treated with a mixture of $5 \%$ bovine serum albumen (BSA)/TBS ( $1 \mathrm{~g}$ Bovine Serum Albumen in $20 \mathrm{ml}$ TBS) for $10 \mathrm{~min}$.

The antibodies were diluted $1: 5$ for anti-CD31, 1:10 for antiCD141, 1:20 for anti-CD34 and 1:1000 for anti-human vWF and Ki67 in 5\% BSA/TBS. The sections were incubated with primary antibodies at room temperature for $40 \mathrm{~min}$. Secondary antibodies were incubated for $40 \mathrm{~min}$ using EnVision Labelled Polymer (mouse or rabbit as appropriate). Sections were washed with TBS between incubation steps. 33'diaminobenzidine, DAB was used as a chromogen. The last two steps were carried out using a commercial kit (DAKO EnVision $\mathrm{TM}^{+}$System, Peroxidase (DAB), USA).

The cell nuclei were counter-stained with Haematoxylin in solution. The sections were dehydrated through four changes of IMS and three changes of xylene before being mounted in resinous mountant (DPX, BDH Laboratory supplies, UK). Omission of the primary antibody was used as a negative control and the microvessels of the normal adjacent breast tissue served as internal positive controls.

\section{Assessment of tumour vascularity}

NB determined vascular density without knowing how the architecture or nuclear grade had been classified. No scanning of stained microvessels to identify 'hot-spots' at low magnification was undertaken. The assessment of completely transected involved ducts started from the upper right of all stained sections, moving downwards and to the left. All or the first 50 foci (duct cross sections) encountered were assessed on each section, thus eliminating selection bias.

In the assessment of DCIS accompanied by invasive carcinoma, only those foci of DCIS that were at least $2 \mathrm{~mm}$ away from the invasive component were evaluated to minimise detecting direct local effects of the invasive disease.

\section{Evaluation of DCIS area}

The area of each individual focus of DCIS was measured at $\times 200$ magnification using an image analysis system (Zeis Axiohome with software version 3.0, Germany). In addition, the area with a circumference $100 \mu \mathrm{m}$ from the edge of an individual focus of DCIS was measured. The area required for MVD assessment was thus calculated by subtracting the former from the latter (Figure 1).

\section{Counting microvessels}

The microvessels within $100 \mu \mathrm{m}$ around each DCIS focus were counted at high magnification $(\times 400)$. Eligible microvessels included any immunostained endothelial cell or cluster of cells around a visible lumen clearly separated from adjacent microvessels, tumour cells and other connective tissue components (Figure 2). The presence of red blood cells was not required. It was not possible to distinguish blood and lymphatic vessels. Where vessels were in clusters, each was counted as separate if it met the above criteria.

\section{Controls}

Normal breast lobules were used as internal controls and up to five normal lobules were assessed for each case of DCIS. The normal lobules were assessed only if they were situated more than $2 \mathrm{~mm}$ from the nearest tumour.

\section{Evaluation of degree of necrosis}

As part of the histological assessment, the degree of necrosis was semi-quantitatively assessed in sections of high grade DCIS from patients with and without invasive disease. Each individual focus of DCIS was given a score of 1-3. A score of 1 was given when no necrosis was present in an individual focus of DCIS, a score of 2 when between 1 and $50 \%$ necrosis was present and a score of 3 when there was more than $50 \%$ necrosis.

The data for each duct space were analysed using the KruskalWallis method and Mann-Whitney $U$-test and by the 2-tailed Spearman's or Pearson's Correlation coefficient using SPSS software (Version 10.0 for Window NT).

\section{RESULTS}

The two groups of DCIS were matched for nuclear grade, each contained 7 low, 6 intermediate and 7 high nuclear grade cases.

\section{Microvessel density in DCIS without invasive carcinoma}

Normal lobules The highest values of MVD were obtained using vWF followed by CD34, CD141 and CD31. The mean MVD was significantly different between antibodies (Mann-Whitney, highest $P=0.01$ ) apart from between CD141 and CD34 (Mann-Whitney, $P=0.28$ ). (Table 1; Figure 3).

DCIS For DCIS the highest MVD was obtained using the antibody to CD34, followed by CD141, vWF and CD31. In comparison to normal lobules there were increases in CD34, CD141 and CD31 positive vessels but a decrease in those immunopositive for vWF. Changes in MVD detected by the CD34, CD31 and vWF antibodies were statistically significant (Mann-Whitney, highest $P=0.015$ ) (Table 1). MVD around DCIS using CD34 was less than that around normal lobules using anti-vWF. However, the difference was not statistically significant (Mann-Whitney, $P=0.35$ ).

\section{Microvessel density in DCIS with invasive carcinoma}

Normal lobules In normal lobules surrounding cases of pure DCIS, MVD was highest when stained with vWF, whereas the 


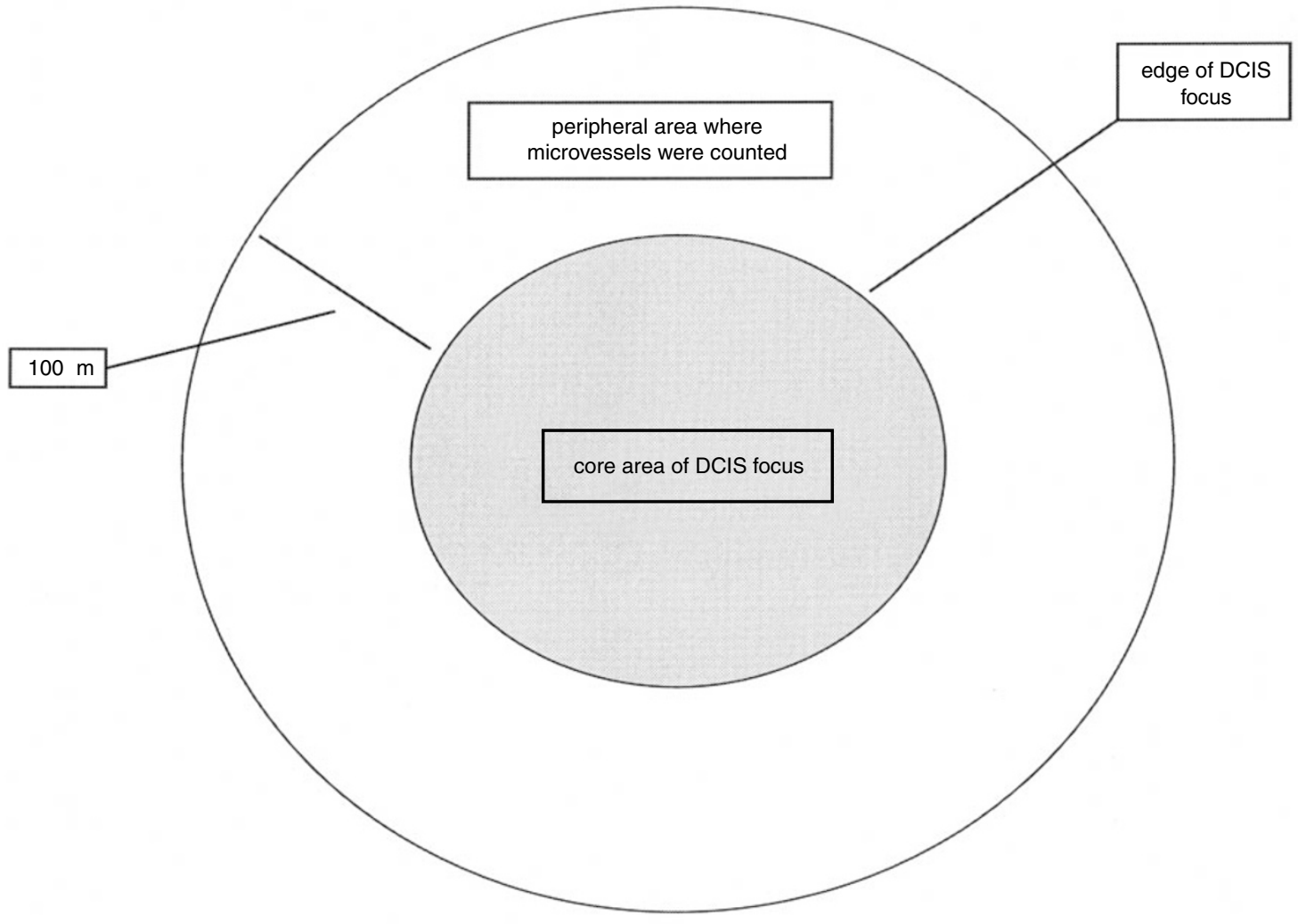

Figure I Simplified diagram of an individual focus of ductal carcinoma in situ (DCIS) and peripheral area where the periductal microvessels were counted.

normal lobules surrounding areas of invasive carcinoma and DCIS had the highest MVD with CD34. However, the only significant differences between the antibodies were that CD31 gave lower values than the other three (Mann-Whitney, highest $P<0.001$ ) (Table 1). The MVD in normal lobules with DCIS and invasive cancer obtained with vWF was significantly lower than in normal lobules from cases of pure DCIS (Mann-Whitney, $P=0.014$ ) whereas those obtained with CD31, CD34 and CD141 showed no significant difference (Mann-Whitney, lowest $P=0.2$ ).

DCIS In DCIS, the highest MVD was obtained using the CD34 antibody. There were significant differences among all four antibodies (Mann-Whitney, highest $P=0.034$ ). (Table 1 ). In DCIS with invasive carcinoma, in common with cases of pure DCIS, there was an increase above normal in CD31, CD34 and CD141 but a decrease in vWF immunopositive vessels. All these changes were statistically significant (Mann-Whitney, highest $P=0.03$ ). When DCIS with invasive cancer compared with pure DCIS, we showed an increase in MVD as determined by CD34 and 141 and decreases as determined by CD31 and vWF. These findings were all statistically significant (Mann-Whitney, highest $P=0.001$ ) except for CD31 (Mann-Whitney, $P=0.3$ ).

\section{Relationship between MVD and nuclear grade}

MVD increased from low to intermediate nuclear grade but decreased in high grade DCIS usually to a level below that of low grade. The difference between low and intermediate grade DCIS was statistically significant for pure and invasive cases using all antibodies (Mann-Whitney, highest $P=0.007$ ) except for pure DCIS when MVD was determined using vWF (Mann-Whitney, $P=0.07)$. The difference between intermediate and high grade was significant for both pure and invasive cases of DCIS with all antibodies (Mann-Whitney, highest $P<0.001$ ) except for invasive cases when MVD was determined by CD31 (Mann-Whitney, $P=0.4$ ). The difference between low and high grade, however, was significant only for pure DCIS using CD141, CD31 and vWF antibodies and for invasive cases using CD31 (Mann-Whitney, highest $P=0.047$ ) (Figure 4).

\section{Relationship between MVD and core area}

For all cases of DCIS the MVD fell with increasing size of the individual focus of DCIS. This negative correlation was statistically significant as determined with all four antibodies (Spearmans, highest $P<0.001$ ) except when CD31 was used on cases of DCIS associated with invasion (Spearmans, $P=0.3$ ) (Figure 5).

\section{Relationship between MVD, core area and necrosis}

As these analyses were restricted to high nuclear grade DCIS, cases with and without invasive carcinoma were pooled. A significant negative correlation was observed between necrosis scores and MVD using all four antibodies (Spearmans, highest $P=0.002$ ) (Figure 6).

A significant positive correlation was also observed between necrosis scoring and core area of the foci for both pure DCIS and DCIS associated with invasion (Spearmans, highest $P<0.001$ ) (Figure 7).

\section{DISCUSSION}

Angiogenesis has been shown to have prognostic significance in breast cancer (Weidner et al, 1991; Bosari et al, 1992; Horak et al, 1992; Weidner et al, 1992) and several studies have shown that pre-malignant lesions of the breast can induce angiogenesis in animal experimental systems and in human breast (Gimbrone and Gullino, 1976; Heffelfinger et al, 1996; Brem et al, 1977; Lich- 
tenbeld et al, 1998). In 1976, Gimbrone et al (1976) showed that $30 \%$ of implants from pre-malignant murine hyperplastic alveolar nodules showed angiogenesis on the irises of New Zealand White rabbits. Furthermore, tissues of higher predicted tumour incidence induced greater neo-vascular responses than did those with a lower probability of malignant transformation. These findings have been confirmed in a number of similar studies (Guinebretiere et al,

A

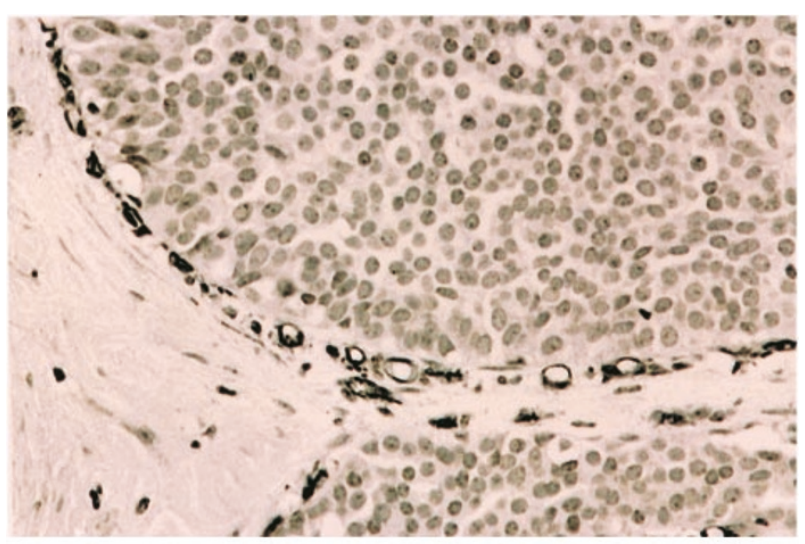

B

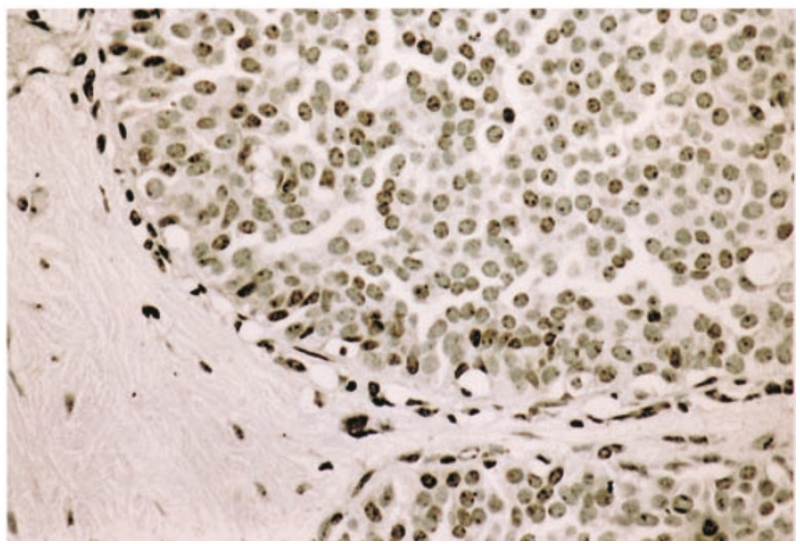

Figure 2 (A) Ductal carcinoma in situ (DCIS) stained immunohistochemically using the CD34 antibody revealing a rim of positive vessels, (B) same area stained for VWF. There is a striking difference in the number of positive cells with the two antibodies in this case.
1994; Brem et al, 1977; Lichtenbeld et al, 1998). We therefore felt that it was reasonable to hypothesize that changes in periductal vascularity may precede the development of invasive carcinoma in DCIS.

A

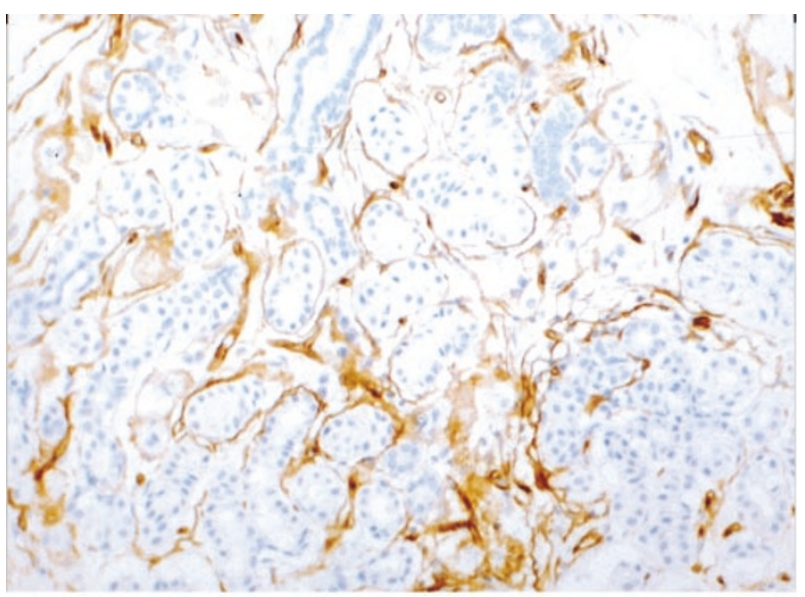

B

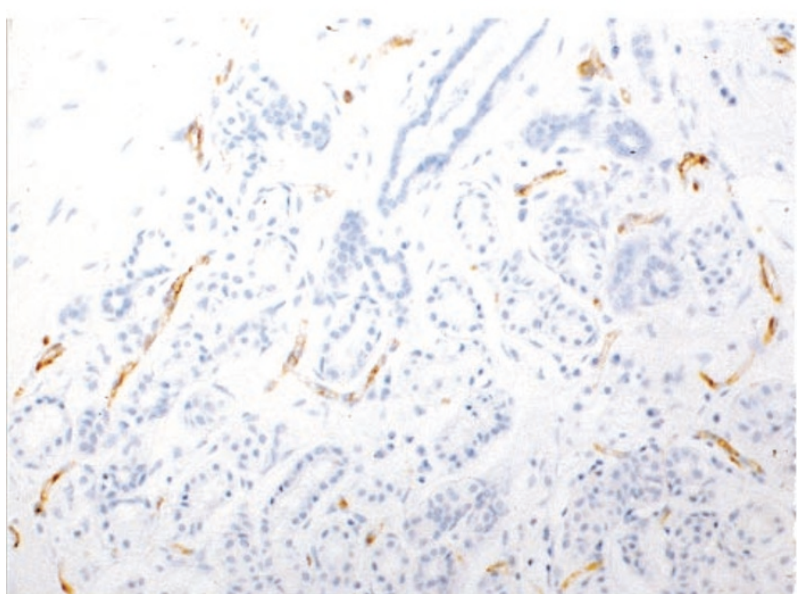

Figure 3 (A) Non-cancerous lobules stained immunohistochemically using the CD34 antibody revealing a rim of positive vessels, (B) same area stained for $v W F$. There is also a striking difference in the number of positive cells with the two antibodies in this case.

Table I The microvessel density (MVD) surrounding normal breast lobules and Ductal carcinoma in situ (DCIS) using four differnet endothelial markers. (s.d.=standard deviation)

\begin{tabular}{|c|c|c|c|c|}
\hline & $\begin{array}{l}\text { MVD stained } \\
\text { with vWF antibody } \\
\text { (vessels } \mathbf{~ m m}^{-2} \text { ) }\end{array}$ & $\begin{array}{l}\text { MVD stained } \\
\text { with CD3I antibody } \\
\text { (vessels } \mathrm{mm}^{-2} \text { ) }\end{array}$ & $\begin{array}{c}\text { MVD stained } \\
\text { with CDI4I antibody } \\
\text { (vessels } \mathrm{mm}^{-2} \text { ) }\end{array}$ & $\begin{array}{c}\text { MVD stained } \\
\text { with CD34 antibody } \\
\text { (vessels } \mathrm{mm}^{-2} \text { ) }\end{array}$ \\
\hline $\begin{array}{l}\text { Normal lobules } \\
\text { (in patients with } \\
\text { Pure DCIS) }\end{array}$ & 106 (s.d. $=74)$ & 37 (s.d. $=58)$ & 80 (s.d. $=77)$ & $86($ s.d. $=69)$ \\
\hline $\begin{array}{l}\text { Normal lobules } \\
\text { (in patients with } \\
\text { DCIS and invasive } \\
\text { carcinoma) }\end{array}$ & 78 (s.d. $=50)$ & 28 (s.d. $=42)$ & 85 (s.d. $=75)$ & 89 (s.d. $=52)$ \\
\hline Pure DCIS & 84 (s.d. $=64)$ & 44 (s.d. $=47)$ & 88 (s.d.=64) & 94 (s.d. $=48)$ \\
\hline $\begin{array}{l}\text { DCIS (with } \\
\text { associated invasive } \\
\text { carcinoma) }\end{array}$ & 68 (s.d. $=54)$ & $4 \mid(s . d .=36)$ & 110 (s.d. $=85)$ & 116 (s.d. $=72)$ \\
\hline
\end{tabular}



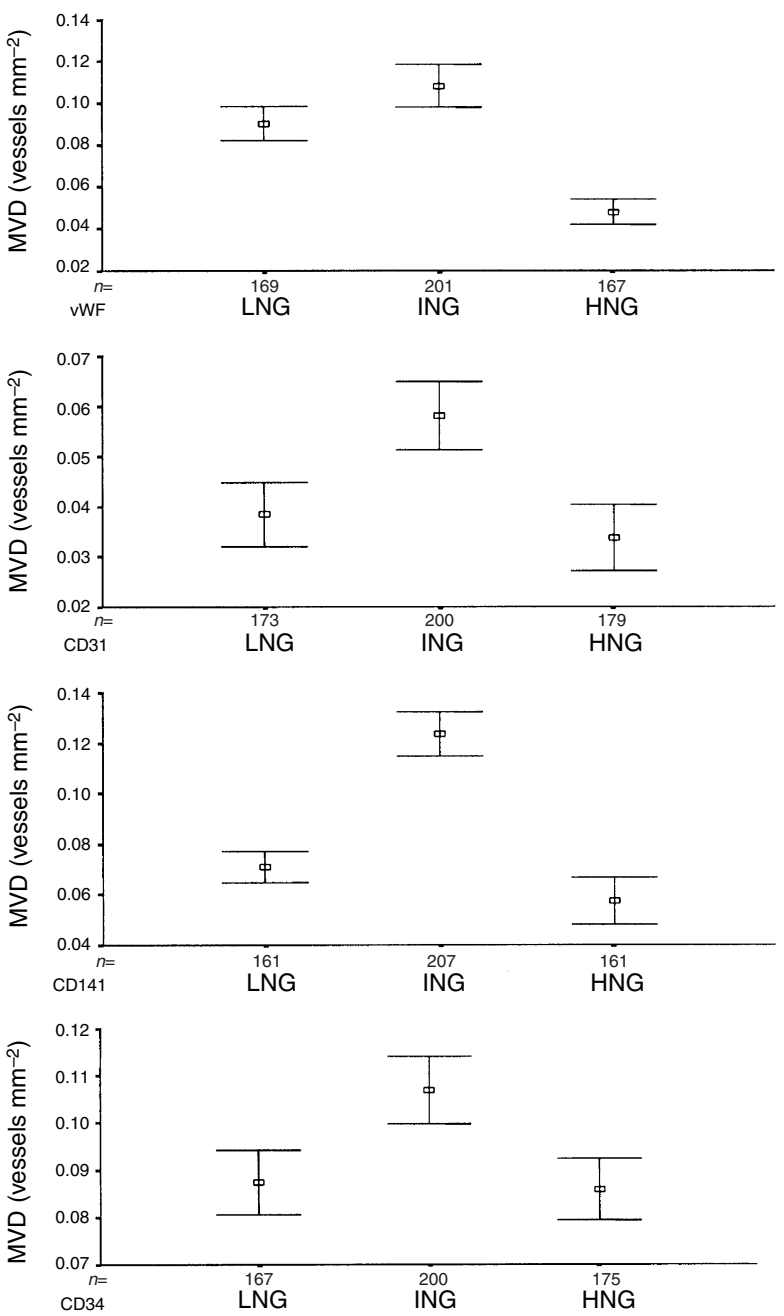

Figure 4 Relationship between microvessel density (MVD) and nuclear grade in ductal carcinoma in situ (DCIS) without invasive carcinoma using four types of endothelial markers. The boxes represent the mean values and the whiskers represent $95 \%$ confidence interval of the mean. (LNG=low nuclear grade, ING=intermediate nuclear grade, HNG=high nuclear grade).

Two patterns of angiogenesis have been described in DCIS: a diffuse stromal vascularity (pattern I) and a vascular rim around the involved ducts (pattern II) (Guidi et al, 1994). Subsequently, it was proposed that pattern II resulted from angiogenic factors secreted by tumour cells and pattern I from recruitment of accessory cells, which release angiogenic factors (Engels et al, 1997a). In our study, we restricted our investigations to vascular pattern II as we hypothesized that incipient invasion is likely to be associated with vascular changes in the immediate vicinity of the tumour cells.

In the present study the different antibodies used gave different values for MVD both in normal lobules and DCIS indicating that they identified different sub-populations of small vessels. In pure DCIS, there was a significant increase above normal in $\mathrm{CD} 34^{+}$ and $\mathrm{CD}_{3} 1^{+}$vessels but a reduction in those staining for vWF. These findings are in keeping with a change in phenotype as well as vascular density.

In DCIS associated with invasive carcinoma, there were significantly greater numbers of $\mathrm{CD} 34^{+}$and $\mathrm{CD} 141^{+}$vessels and fewer staining for vWF compared with pure DCIS. It is not clear whether this higher periductal MVD reflects a greater predisposition to

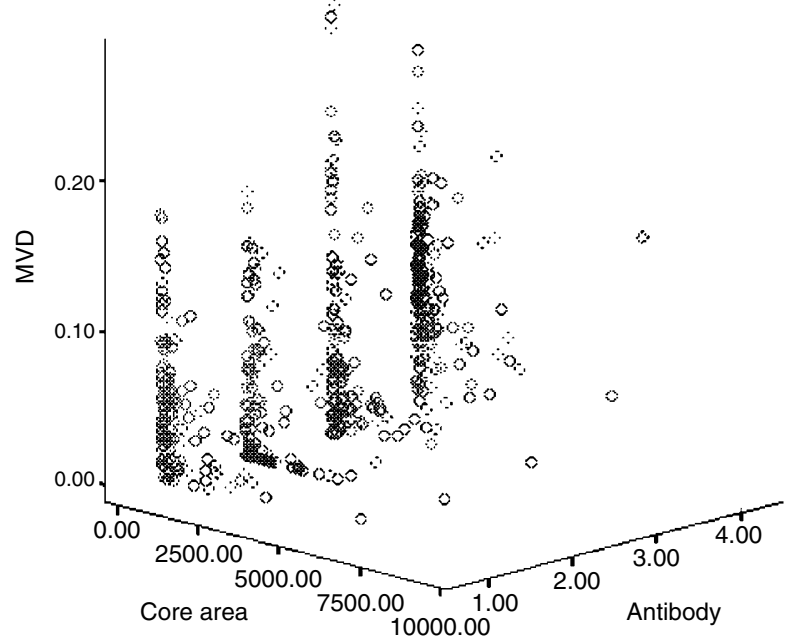

Figure 5 Relationship between microvessel density (MVD) and core area in ductal carcinoma in situ (DCIS) without invasive carcinoma using four types of endothelial markers. ( $=v W F, 2=C D 3|, \quad 3=C D| 4 \mid$, $4=$ CD34).

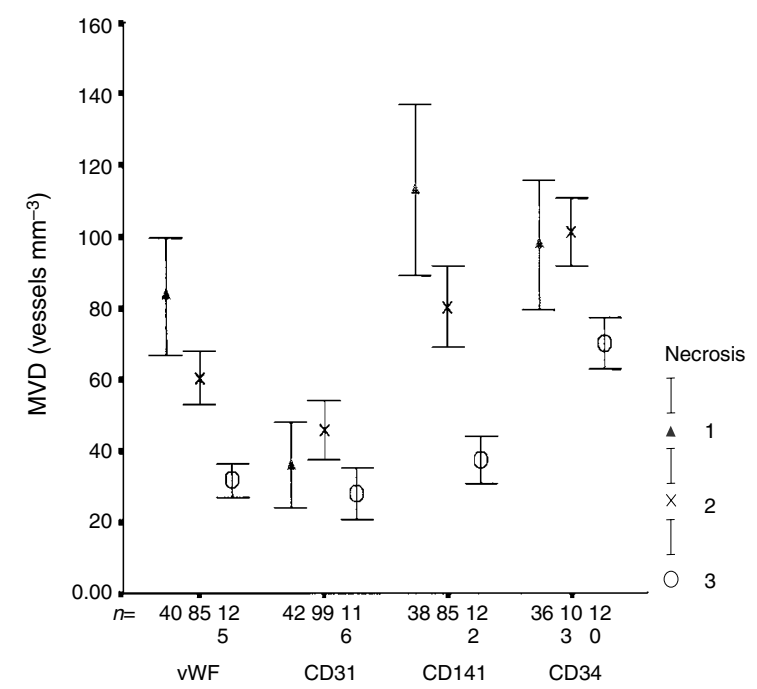

Figure 6 Relationship between microvessel density (MVD) and degree of necrosis in ductal carcinoma in situ (DCIS) with and without invasive carcinoma. The boxes represent the mean values and the whiskers represent 95\% confidence interval of the mean. (Degree of necrosis: (I) no necrosis, (2) $1-50 \%$ of DCIS focus has necrosis, (3) $>50 \%$ of DCIS focus has necrosis).

invade or whether the effect is due to the proximity of invasive carcinoma. We attempted to minimize the latter possibility by evaluating DCIS at least $2 \mathrm{~mm}$ from the nearest invasive carcinoma but this would not exclude any effect of factors released by the tumour into the blood or lymphatic system. It is of interest in this context that the normal lobules from cases of invasive carcinoma exhibited significantly lower numbers of $\mathrm{vWF}^{+}$vessels than those from cases of pure DCIS. This finding is in apparent conflict with the work of Heffelfinger et al (1996) who found the vascularity of histopathologically normal epithelium to be greater in breast containing invasive carcinoma using an antibody to vWF. The breasts without invasive disease in this study, however, included those with benign proliferative change as well as DCIS. 


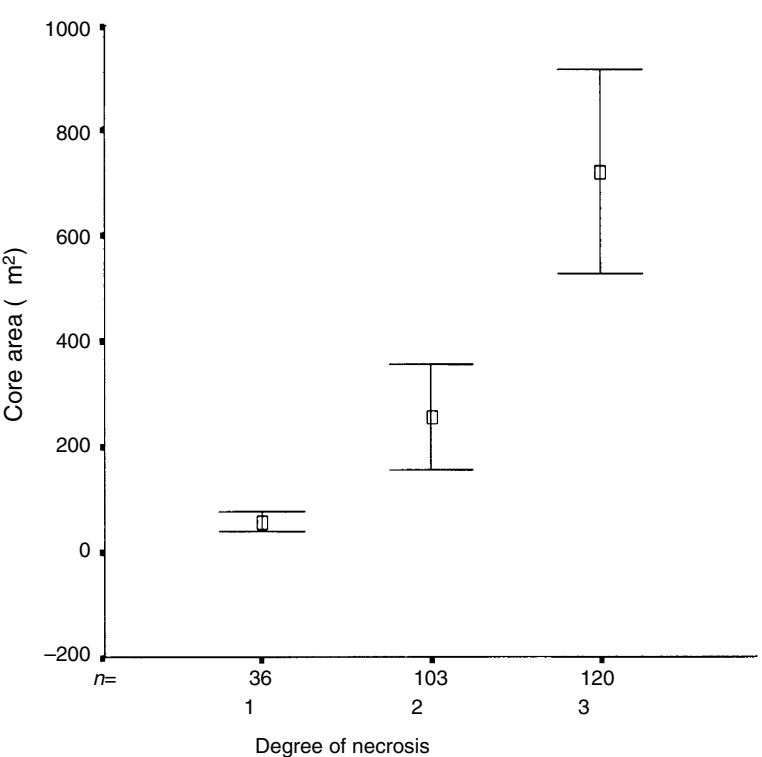

Figure 7 Relationship between degree of necrosis and core area in ductal carcinoma in situ (DCIS) with and without invasive carcinoma. The boxes represent the mean values and the whiskers represent $95 \%$ confidence interval of the mean. (Degree of necrosis: (I) no necrosis, (2) I $50 \%$ of DCIS focus has necrosis, (3) $>50 \%$ of DCIS focus has necrosis).

The different antibodies used gave different values for MVD in normal breast and showed dissimilar changes in DCIS. For patients with and without invasive disease, $\mathrm{vWF}^{+}$vessels were at lower density in DCIS than in the normal breast. This is in keeping with observations on invasive tumours (colorectal carcinoma) where vWF immunostaining has been found to be absent from some of the capillaries in the tumour (Vermeulen et al, 1995). Anti-vWF stains large vessels more strongly than small ones (Vermeulen et al, 1996) and consequently our findings could reflect the immaturity of newly formed tumour-associated vessels. The density of $\mathrm{CD}^{+} 4^{+} \mathrm{vWF}^{-}$or $\mathrm{CD}_{14} 1^{+} / \mathrm{vWF}^{-}$vessels could thus reflect the rate tumour angiogenesis and the consequences it has for the biological behaviour of DCIS.

CD31 gave consistently lower values for MVD than the other antibodies. The molecule has a role in platelet adhesion in inflam- mation and wound healing (Parums et al, 1990) and is expressed on large and small vessels in either normal or tumour tissue (Horak et al, 1992; Toi et al, 1993; Vermeulen et al, 1996). In contrast to vWF it appears not to be expressed on lymphatic vessels (Burgdorf et al, 1981) and this could at least partially explain why determinations of MVD with the CD31 antibody were consistently lower in both normal lobules and DCIS. The possible contribution of lymphatics to neo-vascularisation in tumours has been largely ignored.

Values for MVD as determined using CD34 and 141 antibodies were roughly comparable both in normal lobules and DCIS. Both showed a significant increase in DCIS, with greater values in cases associated with invasive carcinoma. Our findings confirm those of Martin et al (1997), that CD34 antibodies give consistently higher vessel counts in breast carcinomas than antibodies against vWF or CD31.

We found an unexpected relationship between MVD and nuclear grade of DCIS. In those with and without invasive disease, the MVD was significantly higher in intermediate than either low or high-grades. There was also a significant negative correlation between vascular density and both the cross-sectional areas of the ducts involved and the extent of the necrosis of the tumour they contained. The last finding is in apparent conflict with the study of Guidi et al (1994), who found that comedo-type lesions were more likely to be associated with higher microvessel density. In their study, however, vessels up to $500 \mu \mathrm{m}$ from the involved structures were included and only those from the five most vascular fields were recorded. Vessels forming cuffs around ducts were not studied quantitatively as they were found only in a minority of cases. These authors, however, identified vessels using an antibody to vWF only, which in our study stained significantly fewer vessels than the CD34 and CD141 antibodies. A likely explanation for our findings is that angiogenesis increases with growth rate but is unable to keep pace with the most rapidly growing lesions. The rapidly proliferating lesions tended to contain larger foci of DCIS and often showed comedo necrosis.

In conclusion, the greater density of $\mathrm{CD} 34^{+}$and $\mathrm{CD} 141^{+}$vessels around DCIS associated with invasive carcinoma could reflect a greater predisposition to invade but a direct effect of co-existent invasive carcinoma cannot entirely be ruled out in the present study. The relationship between vascular density, grade, duct size and nuclear grade suggests that periductal angiogenesis increases with tumour growth rate but is unable to keep pace with the most rapidly growing lesions.

\section{REFERENCES}

Badve S, S'Hern RP, Ward AM, Millis RR, Pinder SE, Ellis IO, Gusterson BA, Sloane JP (1998) Prediction of local recurrence of ductal carcinoma in situ of the breast using five histological classifications: A comparative study with long follow-up. Hum Pathol 29: 915-923

Bosari S, Lee AKC, DeLellis RA, Wiley BD, Heatley GJ, Silverman ML (1992) Microvessel quantitation and prognosis in invasive breast carcinoma. Hum Pathol 23: 755-761

Brem SS, Gullino PM, Medina D (1977) Angiogenesis: a marker for neoplastic transformation of mammary papillary hyperplasia. Science 195: $880-$ 881

Burgdorf WHC, Mukae K, Rosai J (1981) Immunohistochemical identification of factor VIII-related antigen in endothelial cells of cutaneous lesions of alleged vascular nature. Am J Clin Pathol 75: 167-171

Engels K, Fox SB, Whitehouse RM, Gatter KC, Harris AL (1997a) Distinct angiogenic patterns are associated with high-grade in situ ductal carcinoma of the breast. J Pathol 181: 207-212

Engels K, Fox SB, Whitehouse RM, Gatter KC, Harris AL (1997b) Up-regulation of Thymidine Phosphorylase expression is associated with a discrete pattern of angiogenesis in ductal carcinomas in situ of the breast. J Pathol 182: $414-420$
Fryberg ER, Bland KI (1994) Overview of the biology and management of ductal carcinoma in situ of the breast. Cancer 74: 350-361

Gimbrone Jr MA, Gullino PM (1976) Angiogenic capacity of preneoplastic lesions of the murine mammary gland as a marker of neoplastic transformation. Cancer Res 36:(7PT2): 2611-2620

Guidi AJ, Fischer L, Harris JR, Schnitt SJ (1994) Microvessel density and distribution in ductal carcinoma in situ of the breast. J Natl Cancer Inst 86(8): $614-619$

Guinebretiere JM, Monique GL, Gavoille A, Bahi J, Contesso G (1994) Angiogenesis and risk of breast cancer in women with fibrocystic disease. J Natl Cancer Inst 86: 635-636

Heffelfinger SC, Yassin R, Miller MA, Lower E (1996) Vascularity of proliferative breast disease and carcinoma in situ with histological features. Clin Cancer Res 2(11): $1873-1878$

Horak E, Leek R, Klerk N, Lejeune S, Smith K, Stuart N, Greenall M, Stepniewska K, Harris AL (1992) Angiogenesis, assessed by platelet/ endothelial cell adhesion molecule antibodies, as indicator of node metastases and survival in breast cancer. Lancet 340: 1120-1124 
Lee AHS, Dublin EA, Bobrow LG (1999) Angiogenesis and expression of Thymidine Phosphorylase by inflammatory and carcinoma cells in ductal carcinoma in situ of the breast. J Pathol 187: 285-290

Lee AHS, Happerfield LC, Borrow LG, Millis RR (1997) Angiogenesis and inflammation in ductal carcinoma in situ of the breast. J Pathol 181: $200-206$

Lichtenbeld HC, Barendsz-Janson AF, van Essen H, Struijker Boudier H, Griffeoen AW, Hillen HF (1998) Angiogenic potential of malignant and non-malignant human breast tissues in an in vivo angiogenesis model. Int J Cancer 77: 455-459

Martin L, Green B, Renshaw C, Lowe D, Rudland P, Leinster SJ, Winstanley J (1997) Examining the technique of angiogenesis assessment in invasive breast cancer. Br J Cancer 76(8): 1046-1054

National co-ordinating group for breast screening pathology (1997) Pathology Reporting in Breast Cancer Screening. Sheffield: NHSBSP Publications No 3: $22-27$

Nemoto T, Vana J, Bedwani RN, Baker HW, McGregor FH, Murphy GP (1980) Management and survival of female breast cancer: results of a national survey by the American College of Surgeons. Cancer 45: $2917-$ 2924

Parums DV, Cordell JL, Micklem K, Heryet AR, Gatter KC, Mason DY (1990) JC70: a new monoclonal antibody that detects vascular endothelium associated antigen on routinely processed tissue sections. J Clin Pathol 43: $752-757$

Recht A, Danoff BS, Solin LJ, Schnitt S, Connolly J, Botnick L, Goldberg I, Goodman RL, Harris JR (1985) Intraductal carcinoma of the breast: results of treatment with excisional biopsy and irradiation. J Clin Oncol 3: 13391343

Sales A, Ruiz A, Llombart-Bosch A (1999) Comparative morphometric evaluation of microvessel density and nuclear area in ductal carcinoma in situ and hyperplastic ductal breast lesions. The Breast 8: $21-25$
Silverstein MJ, Barth A, Poller DN, Gierson ED, Colburn WJ, Waisman JR, Gamagami P (1995) Ten-year results comparing mastectomy to excision and radiation therapy for ductal carcinoma in situ of the breast. Eur $J$ Cancer 31: $1425-1427$

The consensus conference committee (1997) Consensus conference on the classification of ductal carcinoma in situ. Cancer 80: 1798-1802

Toi M, Kashitani J, Tominaga K (1993) Tumour angiogenesis is an independent prognostic indicator of primary breast carcinoma. Int J Cancer 55: $371-374$

Valtola R, Slaven P, Heikkila P, Taipale J, Joensuu H, Rehn M, Pihlajanieme T, Weich H, de Waal R, Alitalo K (1999) VEGFR-3 and its ligand VEGF-C are associated with angiogenesis in breast cancer. Am J Pathol 154(5): $1381-1390$

Vermeulen PB, Gasparini G, Fox SB, Toi M, Martin L, McCulloch P, Pezzella F, Viale G, Weidner N, Harris AL, Dirix Y (1996) Quantification of angiogenesis in solid human tumors: an international consensus on the methodology and criteria of evaluation. Eur J Cancer 32A:(14): 2474-2484

Vermeulen PB, Verhoeven D, Fierens H, Hubens G, Goovserts G, Van Marck E, De Bruijn EA, Van Oosterom ??, Dirix LY (1995) Microvessel quantification in primary colorectal carcinoma: an immunohistochemical study. $\mathrm{Br} J$ Cancer 71: $340-343$

Weidner N, Folkman J, Pozza F, Bevilacqua P, Allred N, Moore DH, Meli S, Gasparini G (1992) Tumour angiogenesis: a new significant and independent prognostic indicator in early-stage breast carcinoma. J Natl Cancer Inst 84: $1875-1887$

Weidner N, Semple JP, Welch WR, Folkman J (1991) Tumour angiogenesis and metastasis-correlation in invasive breast carcinoma. N Engl J Med 324: $1-8$ 